\title{
Gute Qalys, schlechte Qalys?
}

Das SGB V kennt in seiner aktuellen Fassung eine Reihe von Ausnahmen in Bezug auf die Erstattungsfähigkeit von Arzneimitteln. Diese beziehen sich unter anderem auf sog. "Lifestyle"-Präparate. Zu dieser Gruppe zählende Arzneimittel weisen z. T. ein äußerst vorteil-haftes Kosten-Nutzwert-Verhältnis auf. Die Kriterien, die dem Ausschluss bestimmter Arzneimittel zugrunde liegen, sind unklar und nicht nachvollziehbar. Der vorliegende Beitrag analysiert vor dem Hintergrund einer wachsenden Bedeutung des so genannten Health Technology Assessment die Ausnahmeregelungen des SGB V aus einer gesundheitsökonomischen Perspektive.

\section{Matthias Staat}

\section{Einleitung}

In einer größer werdenden Zahl von Ländern wird auf Verfahren des sogenannten Health Technology Assessment (HTA) zurückgegriffen, um insbesondere neue Methoden und Arzneimittel einer Überprüfung im Hinblick auf deren Wirksamkeit und Wirtschaftlichkeit zu unterziehen. Diese Entwicklung kann vor dem Hintergrund der Finanzierungsprobleme im Gesundheitswesen gesehen werden; sie lässt allerdings ebenso auf eine Tendenz zu einer genaueren Prüfung der Kosten-Nutzen-Relation mit dem Ziel der Verbesserung von Allokationsentscheidungen im Gesundheitswesen - unbeschadet von Finanzierungsfragen - schließen.

Die Methoden der Wissensbewertung im Rahmen von HTA beruhen auf denjenigen der Evidenzbasierten Medizin (EbM). Den Beginn der EbM, wie sie heute verstanden wird, geht auf den britischen Epidemiologen Cochrane zurück. Erkenntnisse der EbM werden von einem internationalen Netzwerk von Wissenschaftlern in der sogenannten Cochrane Library gesammelt und publiziert. Obwohl es keine einheitliche Definition der EbM gibt, kann als deren zentrales Ziel die Verbesserung der medizinischen Praxis durch das systematische Aufbereiten von Erkenntnissen aus der Forschung angesehen werden. Von dieser die Arzt-Patienten Ebene betreffenden Form der EbM unterscheidet sich die evidenzbasierte Health Care, die sich auf Systementscheidungen bezieht. Insbesondere sollen hierdurch der willkürliche Einsatz therapeutischer Verfahren ohne gesicherte Wirkung vermieden und die Fokussierung auf Verfahren, deren Wirkung zweifelsfrei belegt ist, gefördert werden. Gleichzeitig soll auf diese Weise verhindert werden, dass neue Erkenntnisse nur mit großer Verzögerung in die allgemeine

PD Dr. Matthias Staat, Universität Mannheim, Abteilung Volkswirtschaftslehre
Praxis umgesetzt werden. Durch den zielgerichteten Einsatz verfügbarer Verfahren kann die Allokation der vorhandenen Mittel verbessert werden.

Das Konzept der QALYs (qualitätsbereinigte Lebensjahre, vgl. etwa Dolan, 2000) ist das zentrale Maß für die Bewertung von Verfahren und Arzneimitteln in einer Reihe von Ländern (Australien, England und Wales, Neuseeland, Holland, Norwegen und Schweden; vgl. Kulp und Greiner, 2006). Es stellt auf die sog. Kosten-Nutzwert-Analyse $\mathrm{ab}$ und unterscheidet sich von alternativen Betrachtungsweisen, bspw. der Kosten-Nutzen-Analyse, die eine Bewertung in Geldeinheiten vornimmt, sowie von der Kosten-Effektivitäts-Analyse, die auf klinische Endpunkte rekurriert.

Ein QALY entspricht einem (fiktiven) Lebensjahr bei vollständiger Gesundheit, wobei der Zustand „tot" einem Wert von null gleichgesetzt wird. Eine Anwendung dieses Maßes impliziert, dass man bereit ist, ein Lebensjahr bei vollständiger Gesundheit zweien der Qualität mit 0,5 oder zehn Jahren, deren Qualität mit 0,1 bewertet wird, gleichzusetzen. Ebenso wird eine Steigerung - und um diese geht es letztlich bei der Gesundheitsversorgung um 0,1 QALYs unabhängig davon, ob es sich um die vollkommene Genesung (für die Dauer eines Jahres) ausgehend von einem Wert von 0,9 oder um eine Steigerung ausgehend vom Wert null handelt, gleich bewertet. Insbesondere ist die Bewertung von Veränderungen von Gesundheitszuständen durch QALYs nicht davon abhängig, ob diese durch eine Verlängerung des Lebens bei gegebener Qualität oder durch eine Steigerung der Qualität des gesundheitlichen Zustands ohne gleichzeitige Verlängerung des Lebens erzeugt werden.

\section{Kosten-Nutzwert-Analysen als Instrument der Allokation im Gesundheitswesen}

Eine Kosten-Nutzwert-Analyse bewertet die Wirtschaftlichkeit medizinischer Verfahren oder Arzneimittel nach den Kosten, die pro gewonnenem QALY anfallen. Die 
mit diesem Maß verknüpften Probleme sind vielfältig: angefangen bei der Nicht-Eindeutigkeit bei der Bewertung der „Qualität“ bestimmter Gesundheitszustände was die Vergleichbarkeit entsprechender Studien partiell einschränkt - bis hin zu der Notwendigkeit, Nutzen, die bei verschiedenen Personen anfallen, gegeneinander aufzurechnen, wenn man auf Basis von QALYs Allokationsentscheidungen im Gesundheitswesen fällen möchte. Aufgrund der genannten und weiterer Einschränkungen ist es auch im Idealfall nicht möglich, vollständig wertfreie Urteile in Bezug auf die (wirtschaftliche) Vorteilhaftigkeit einzelner Verfahren und Präparate zu fällen. Dennoch wäre bei einem begrenzten Budget die (theoretisch) optimale Allokationsregel, in aufsteigender Reihenfolge der Kosten pro QALY solange Leistungen zu finanzieren, bis das gegebene Budget vollständig ausgeschöpft ist. Auf diese Weise wird die Summe der mit diesem Budget zu erzeugenden QALYs, die im Rückgriff auf das utilitaristische Wohlfahrtskonzept als Maßstab für den erzeugten Nutzen gelten kann, maximiert. Eine weitere Steigerung des gesellschaftlichen Nutzens würde zusätzliche Mittel erfordern, die allerdings nur solange zu rechtfertigen wären, wie die unterstellte Zahlungsbereitschaft die damit verbundenen Kosten noch deckt.

Die äußerst aufwändige Methodik, die das Berechnen von QALYs bedingt, lässt sich nur dann rechtfertigen, wenn von der zu betrachtenden Leistung eine merkliche Budgetwirkung zu erwarten ist. Dieses Prinzip gilt für Kosten-Nutzen-Analysen in allgemeiner Form (Arrow et al. 1996). Die Durchführung einer Bewertung setzt daher voraus, dass eine solche Budgetwirkung von einer zu untersuchenden Methode ausgeht oder ausgehen wird. Bei der Erstellung entsprechender HTA-Berichte durch Institutionen wie dem NICE (in England und Wales; diesem Institut kommt im Bereich HTA international eine Vorreiterrolle zu) bzw. dem DAHTA (in Deutschland) wird in der Regel ohnehin auf bereits publizierte Studien zurückgegriffen. Soweit Primärstudien bzw. HTA-Berichte nicht für Deutschland erstellt wurden ist zu beachten, dass auf das Ausland bezogene Analysen nur unter bestimmten Prämissen auf Deutschland übertragen werden können (vgl. Kulp und Greiner, 2006).

\section{Problematische Begründung des Ausschlusses von „Lifestyle"-Präparaten}

Vor dem Hintergrund einer Hinwendung zu den Techniken des HTA bedarf es demnach lediglich zweierlei Rahmensetzungen, um eine optimale Allokation der Mittel im Gesundheitswesen zu erreichen: einerseits eine Festlegung auf einen bestimmten Budgetumfang bzw. eine Obergrenze für die Kosten pro QALY und gleichzeitig eine Definition dessen, was als zu behandelnder Krankheitszustand im Sinne des Gesetzes gelten kann. ${ }^{1}$

Das SGB V beinhaltet seit 2004 eine Reihe von Einschränkungen im Hinblick auf die Erstattungsfähigkeit von Arzneimitteln, die auch als „Lifestyle“-Arzneimittel apostrophiert werden $(\$ 34)$. Diese verdienen gerade deshalb besondere Beachtung, weil das Ausgabenvolumen, das nach Schätzungen durch die betreffende Regelung jährlich eingespart werden kann, keine auffällige Größenordung erreicht. Die Gesamtausgaben der GKV für Lifestyle-Präparate beliefen sich laut IGES et al. (2006), Abschnitt 3.4.6, S. 63, im Jahre 2003 insgesamt auf „nur einen geringfügigen Anteil an den Arzneimittelausgaben der GKV“. ${ }^{2}$ Falls der Gesetzgeber an dieser Stelle die Richtung im Hinblick auf Kriterien vorgibt, nach der künftig - etwa aufgrund der Forderung der Beitragssatzstabilität notwendig werdende weitere Ausschlussregelungen zur Erstattung vorgenommen werden sollen, die sich aus den der HTA zugrunde liegenden Kosten-Nutzen-Erwägungen nicht zwingend ableiten lassen, stellt sich die Frage, welche Kriterien dies sein könnten.

Zum Gesetzestext bemerken IGES et al. (2006), S. 390, Fußnote 1, in ihrem Gutachten für das BMG: „Der Gesetzgeber liefert hierzu in $\$ 34$ (1) Satz 7 allerdings eine äußerst problematische Begründung: Von der Versorgung sind außerdem Arzneimittel ausgeschlossen, bei deren Anwendung eine Erhöhung der Lebensqualität im Vordergrund steht. Auf die Erhöhung der Lebensqualität, die einen integralen Bestandteil der gesundheitlichen Outcomes bildet, zielt doch letztlich die gesamte medizinische Versorgung und damit gerade auch die Arzneimitteltherapie." Unabhängig von der spezifischen Begründung, mit der bestimmte Mittel von der Erstattung ausgeschlossen bleiben, gilt, dass ein Ausschluss von Arzneimitteln oder Technologien, deren Wirksamkeit erwiesen ist, zu einem Wohlfahrtsverlust führen kann. Dies ist dann der Fall, wenn die Kosten im Vergleich zum erzeugten Nutzen nicht in einem schlechteren Verhältnis als bei anderen Wirkstoffen, die erstattet werden, stehen.

An dieser Stelle sei angemerkt, dass sich auch die Tatsache, dass durch den Einsatz von Lifestyle-Präparaten ein reiner Gewinn an Lebensqualität, nicht aber von Lebensdauer erzielt würde, prinzipiell nicht als Ausschlusskriterium eignet. Eine reine Steigerung der Lebensqualität ohne eine das Leben verlängernde Wirkung ist ebenso charakteristisch für die Therapie (chronischer) Schmerzen. Sollte der Gesetzgeber hier eine (vermeintlich klare) Trennlinie zwischen einem Gewinn an Lebenszeit und einem Gewinn an reiner Lebensqualität gezogen haben, so lässt sich diese Trennlinie aus der Logik der KostenNutzwert-Betrachtung mittels QALYs nicht ableiten: es gibt keine unterschiedlichen Qualitäten von QALYs, eben gute oder schlechte, je nachdem auf welche Art oder mit welchen Mitteln sie erzeugt werden.

Im Folgenden soll auf die vom § 34 erfassten Präparate zur Raucherentwöhnung näher eingegangen werden. Diese werden im \$ 34 zusammen mit Mitteln zur Behandlung der erektilen Dysfunktion, zur Förderung des Haarwuchses sowie Appetithemmern in eine Klasse von Lifestyle-Präparaten zusammengefasst. Der Umstand, dass der Ausschluss von Mitteln auf Basis des Therapiezwecks erfolgt, ist problematisch. Nur wenn festgestellt 
werden kann, es handele sich bei den betroffenen Diagnosen nicht um Krankheiten im eigentlichen Sinne, wäre ein Ausschluss auf Dauer zu begründen; mit Ausnahme der Mittel zur Förderung des Haarwuchses lassen sich aber alle unter $\S 34$ ausgeschlossenen Mittel zur Therapie von als behandlungsbedürftig eingestuften Zuständen einsetzen.

Ebenso bleibt festzuhalten, dass der Ausschluss der betreffenden Präparate aufgrund des Kenntnisstandes des medizinisch Möglichen bei Einführung der Regelung des § 34 erfolgte. Sollten nun neue Mittel oder Verfahren zur Verfügung stehen, die denjenigen, die zum Zeitpunkt der erstmaligen Verabschiedung der Regelung verfügbar waren, überlegen sind, kann eine automatische Ausweitung der Ausnahmeregelung auf neuere Wirkstoffe zu einer Fehlallokation von Ressourcen führen, selbst wenn dies für die ursprüngliche Liste nicht der Fall gewesen wäre. Zudem weist bereits Weisbrod (1991) auf die Rückwirkungen von Erstattungsregelungen auf die medizinische Forschung hin: ein Wegfall der Erstattung verhindert unter Umständen das Erforschen viel versprechender Wirkstoffe, deren vorteilhaftes Kosten-Nutzen-Verhältnis ihre Entwicklung aus ökonomischer Sicht als wünschenswert erscheinen ließe.

\section{Mögliche Vermeidung künftiger Kosten unter- bleibt}

Die Argumentationslinie, dass der Einsatz der entsprechenden Arzneimittel eine reine Steigerung des Lebensqualität mit sich bringt, lässt sich für die Präparate zur Raucherentwöhnung ohnehin nicht aufrechterhalten. Zusätzlich zu der oben angesprochenen Gefahr der Fehlallokation bei Verfügbarkeit neuer, den herkömmlichen überlegener Wirkstoffe kann der Versuch, heute mit Blick auf die Beitragssatzstabilisierung Einsparungen zu realisieren, dazu führen, dass in Zukunft höhere und eigentlich vermeidbare Kosten auf das Gesundheitssystem zukommen. Die Sorge, dass eine Fokussierung auf die Stabilisierung der Beiträge in der laufenden Periode langfristig Mehrkosten induzieren könnte, spiegelt sich im Gutachten des Sachverständigenrats für die Konzertierte Aktion im Gesundheitswesen (2003), Band II, Ziffer 694, S. 510, wider: „Die auf das Budgetjahr bezogene Beitragssatzstabilität fördert unbeschadet ihrer fiskalischen Bedeutung eine kurzfristige Betrachtungsweise und diskriminiert damit auch investive Projekte, die zwar heute relevante Kosten verursachen, aber künftig erhebliche Erträge versprechen." Waren diese Ausführungen auf eine mögliche Vernachlässigung von Primärprävention bezogen, lässt sich zeigen, dass dies ebenso auf die Raucherentwöhnung zutrifft.

Die jährlichen, durch das Rauchen verursachten Kosten werden in einer Reihe von Studien mit zum Teil voneinander abweichenden, aber ausnahmslos nennenswerten Beträgen beziffert. Die Mehrzahl der Studien summieren direkte und indirekte Kosten, wobei unter den direkten Kosten im Wesentlichen der Ressourcenverzehr für die Behandlung zu verstehen ist, also die Kosten für Therapie, Rehabilitationsmaßnahmen, Arzneimittel, Heil- und Hilfsmittel sowie die bei der Pflege anfallenden Kosten; indirekte Kosten beziehen sich auf ausgefallene Erwerbszeiten.

Bei allen im Folgenden aufgeführten Kostendaten ist zu beachten, dass es sich um periodenbezogene Aussagen für bestimmte, in der Vergangenheit liegende Jahre handelt. Die Folgekosten des Rauchens in Deutschland werden aber in den kommenden Jahren weiter ansteigen (Peto et al., 2006), da vor allem das Rauchen unter Jugendlichen bis in die jüngste Zeit hinein (vgl. Ruff et al., 2000) zunahm. Aufgrund der langen Latenzzeiten bis zum Ausbruch der typischen, durch das Rauchen bedingten Krankheiten, wird der Höhepunkt der Manifestation dieser Folgekrankheiten und damit der Kostenentwicklung erst in Zukunft erreicht werden.

Die erste für Deutschland durchgeführte Studie (Ruff et al., 2000) beziffert die gesamten durch das Rauchen verursachten Kosten für das Jahr 1996 bereits auf 16,6 Mrd. € (Diskontfaktor $4 \%$ ), die sich etwa hälftig auf direkte und indirekte Kosten aufteilen. Hierbei wird die Untersuchung auf die häufigsten Folgeerkrankungen des Rauchens beschränkt. Wegner et al. (2004) kommen allein für die Produktivitätsausfallkosten für das Jahr 1999 auf einen Wert von 14,48 Mrd. € (Diskontsatz 5 \%), wobei die Autoren das Spektrum der betrachteten Folgeerkrankungen des Rauchens gegenüber der Studie von Ruff et al. (2000) erheblich erweitern.

Weder in der Studie von Ruff et al. (2000) noch in derjenigen von Wegner et al. (2004) sind jedoch die Folgekosten, die bspw. aus der Schädigung von Kindern durch das Passivrauchen resultieren, eingerechnet. Diese Kosten sind naturgemäß nur schwer zu beziffern; sie sind in den Berechnungen der Studien, die in dieser Arbeit zitiert werden, generell nicht berücksichtigt. Ein Anhaltspunkt lässt sich einer Arbeit von Fritjers et al. (2006) entnehmen. Sie legen eine Schätzung der so verursachten Kosten vor, die auf einer repräsentativen Stichprobe von ca. 6.000 Kindern in England fußt. Die Kostenabschätzung für die Effekte des Passivrachens wird wie folgt umgesetzt: da zwischen dem Haushaltseinkommen und dem Gesundheitsstatus eines Kindes ein starker, positiver $\mathrm{Zu}$ sammenhang besteht, lässt sich berechnen, um wie viel höher das Haushalteinkommen für ein gegebenes, passiv rauchendes Kind sein muss, damit es denselben Gesundheitsstatus aufweist, wie im Zustand ohne Passivrauchen. Eine obere Grenze für diesen Wert wird von den Autoren mit 30.000 US \$ pro Jahr angegeben.

Aufgrund dieser nennenswerten, durch das Rauchen verursachten Kosten, ließe sich der Einsatz von erheblichen Mitteln rechtfertigen, solange eine effektive Therapie zur Raucherentwöhnung zu Verfügung steht. Law/ Tan (1995) bieten einen Übersichtsartikel über nahezu 200 Studien zur Raucherentwöhnung und kommen zu 
dem Ergebnis, dass die medikamentöse sowie die Nikotinersatztherapie (NRT) die effektivsten unter den untersuchten Alternativen sind. Insbesondere erweisen sich diese Therapiealternativen einer reinen Motivation von Rauchern zur Entwöhnung in Form eines ärztlichen Gesprächs oder durch Broschüren und sonstigem Informationsmaterial unter Verzicht auf diese Wirkstoffe als überlegen. Dieses Ergebnis wurde im Rahmen einer von Fiore et al. (2000) durchgeführten Metaanalyse für den Wirkstoff Bupropion sowie für die NRT bestätigt. Hierbei ist zu beachten, dass die beiden zuletzt genannten Studien die neuesten verfügbaren Wirkstoffe nicht einschließen. Cahill et al. (2007) berichten in der bereits einleitend erwähnten Cochrane Library, dass der neue Wirkstoff Vareniclin eine doppelt so hohe odds ratio $(3,22$ gegenüber $1,6)$ für eine einjährige Abstinenz aufweist wie bereits verfügbare Präparate.

An dieser Stelle wird das bereits angesprochene Problem des Ausschlusses von Präparaten aufgrund ihrer therapeutischen Indikation noch einmal deutlich: Neuere Wirkstoffe werden, unabhängig von ihrer Wirksamkeit und ihres Nutzen-Kosten-Verhältnisses, gegebenenfalls automatisch auch dann aus der Erstattung ausgeschlossen, wenn sich diese den bereits verfügbaren therapeutischen Möglichkeiten als überlegen erweisen sollten.

Verschiedene Studien untersuchen die relative Wirksamkeit unterschiedlicher Designs von Programmen der Raucherentwöhnung im Hinblick auf die bisher verfügbaren Wirkstoffe. Hierbei erweist sich die ärztlich begleitete Gabe von Bupropion oder NRT anderen Designs regelmäßig als überlegen (für einen Überblick vgl. Rasch und Greiner, 2006). Für Deutschland vergleichen Twardella/Brenner (2007) vier Designs, denen Praxen und Patienten, die zuvor mindestens 10 Zigaretten pro Tag konsumierten, nach dem Zufallsprinzip zugeordnet wurden. Den Erfolgsraten der derzeit gängigen Therapiepraxis für eine Abstinenz 12 Monate nach der Behandlung werden die Erfolgsraten folgender Vorgehensweisen gegenübergestellt: mit einer Information der beteiligen Ärzte werden (i) ein finanzieller Anreiz für den Arzt im Falle einer erfolgreichen Entwöhnung, (ii) die Übernahme der Kosten für die Patienten (iii), eine Kombination aus (i) und (ii) verglichen. Es zeigt sich, dass insbesondere bei der Übernahme der Kosten für die Arzneimittel signifikant bessere Erfolge erzielt werden, während eine alleinige Incentivierung der Ärzte nicht zu einer höheren Rate erfolgreicher Entwöhnungen führt. Gleichzeitig rekrutieren die Ärzte deutlich mehr Teilnehmer für das Entwöhnungsprogramm, sobald sie in der Lage sind, eine kostenfreie Therapie anzubieten.

Die Effektivität der bereits verfügbaren Wirkstoffe müsste nicht zu prohibitiven Kosten erkauft werden: Song et al. (2002) geben die Kosten pro QALY für verschiedene medikamentöse Therapien zur Raucherentwöhnung mit 681 bis 2101 US \$ an. Zum Vergleich: das NICE gibt als Größenordnung für akzeptable Kosten pro QALY einen Rahmen von 20.000 bis $30.000 €$ (knapp
60.000 US \$) an, wobei Devlin/Parkin (2003) in einer empirischen Untersuchung der Entscheidungen des NICE zu dem Ergebnis kommen, dass die tatsächliche Schwelle etwas höher als die offiziell angegebene liege. Konsequenterweise kommt das NICE in seiner 2002 veröffentlichten „Guidance on the use of nicotine replacement therapy and bupropion for smoking cessation" zu dem Schluss, dass es sich bei der medikamentösen Raucherentwöhnung um eine der kosteneffektivsten medizinischen Maßnahmen überhaupt handele. Eine Studie von Lightwood/Glantz (1995) kommt allein anhand der Analyse der durch das Rauchen verursachten Folgekosten aufgrund von Herzinfarkten sowie Schlaganfällen in den USA zu dem Schluss, dass eine Raucherentwöhnung Nettoersparnisse für das dortige Gesundheitssystem insgesamt mit sich brächte - die Ersparnis im Zeitraum von sieben Jahren durch eine Senkung des Raucheranteils in der Bevölkerung um $1 \%$ für fünf Jahre wird mit 3,2 Mrd. US \$ beziffert. Gleichzeitig wird die Entwöhnung erwachsener Raucher als kosteneffektiver als alle Präventionsprogramme für Jugendliche eingestuft.

Hervorzuheben ist, dass sich Therapieansätze, die auf den Einsatz von Wirkstoffen verzichten, sowohl von den reinen Erfolgschancen einer dauerhaften Entwöhnung als auch unter Kosten-Nutzen-Aspekten als unterlegen erweisen. Daher ist die Tatsache, dass die reine Beratung / Motivation von Rauchern mit dem Ziel, das Rauchen einzustellen, erstattungsfähig ist, kritisch zu bewerten. Zum einen kann Sie entwöhnungswilligen Rauchern den Eindruck vermitteln, diese Formen von Interventionen würden gerade deshalb erstattet, weil es sich hierbei um die wirksamsten Möglichkeiten der Raucherentwöhnung handele; dies ist nicht zutreffend. Zum anderen bedingt der Einsatz von Mitteln der Kassen für Interventionen mit relativ geringer Wirksamkeit eine eigentlich vermeidbare Fehlallokation und somit höhere Kosten für das Gesundheitswesen. Zudem birgt die Möglichkeit, für die nicht verschreibungspflichtige NRT Werbung zu betreiben, während dies für verschreibungspflichtige Wirkstoffe nicht möglich ist, die Gefahr private Mittel in diese Therapieform zu lenken; auch dies resultiert ggf. in einer Fehlallokation.

Aus Kostensicht ist somit nicht zu erklären, weshalb die Erstattung von Mitteln zur Raucherentwöhnung per Gesetz ausgeschlossen wird. Mittel- bis langfristig wären gegebenenfalls substanzielle Einsparungen durch den verstärkten Einsatz von Mitteln zur Raucherentwöhnung zu realisieren. Hierbei erhöht die Kostenerstattung für den Patienten nach heutigen Erkenntnissen nicht nur die Zahl der Raucher, die eine Anstrengung zur Entwöhnung unternehmen, sondern auch die Erfolgswahrscheinlichkeit eines Entwöhnungsversuchs signifikant. Daneben sind auch „Mitnahmeeffekte“ durch im medizinischen Sinne nicht Bedürftige, wie sie bei anderen, als Lifestyle-Präparate eingestuften Wirkstoffen unterstellt werden könnten, bei Arzneimitteln zur Raucherentwöhnung nicht zu vermuten. ${ }^{3}$ 
Auch wenn man die Gesundheitsversorgung nicht auf das Abarbeiten sog. league tables, also Aufstellungen in Form einer „QALY-Bestenliste“, reduzieren möchte, geben die möglichen zukünftigen Kostenfolgen der in $§ 34$ geschaffenen Ausnahmetatbestände zu denken. Eine stringente und nachvollziehbare Begründung der einzelnen Ausnahmen wäre notwendig. Eine Kasuistik von Ausnahmetatbeständen erschwert es Patienten, sinnvolle von nicht sinnvollen Behandlungsmöglichkeiten zu trennen und verhindert ebenso, dass diese eine Einschätzung darüber erlangen, welche Leistungen das öffentliche Gesundheitswesen in Zukunft bereithalten wird und welche nicht. Gleichzeitig wäre darauf zu achten, dass eine einmal begründete Liste nicht ohne Berücksichtigung neuer Entwicklungen und Erkenntnisse automatisch fortgeschrieben wird; dies birgt die Gefahr, dass Therapiealternativen Patienten auch dann nicht zur Verfügung stehen, wenn diese den Möglichkeiten, die zum Zeitpunkt der erstmaligen Festlegung des Ausschlusses verfügbar waren, gegebenenfalls weit überlegen sind.

Darüber hinaus steht die Nicht-Erstattung von Mitteln zur Raucherentwöhnung im Gegensatz zu verschiedenen grundlegenden Prinzipien des SGB V wie der Verminderung sozialer Ungleichheiten im Bezug auf Gesundheitschancen sowie der Vermeidung der Gefährdung der gesundheitlichen Entwicklung von Kindern. Auf diese Aspekte soll abschließend eingegangen werden.

\section{Aspekt der Verminderung sozialer Ungleichheit bleibt unberücksichtigt}

„Gesundheitliche Ungleichheit zu beseitigen war und ist ein politisches Ziel der Bundesregierung. Die Ergebnisse des Projektes bestätigen, dass Deutschland auf einem guten Weg ist, Prävention und Gesundheitsförderung so auszubauen, dass sozial Schwächere erreicht werden. Hierzu bedarf es der Kooperation aller Akteure", führt Dr. Klaus Theo Schröder, Staatssekretär im BMG, in einem Kommentar zum Projekts „Closing the Gap“, zu gesundheitlichen Ungleichheiten in Europa aus. ${ }^{4}$ In derselben Pressemitteilung wird zum Thema Rauchen und soziale Ungleichheit festgestellt: „Schulbildung und Rauchen hängen eng zusammen, insbesondere in jüngeren Altersgruppen. Während 60 \% der Männer mit Hauptschulabschluss rauchen, sind es bei Abiturienten $35 \%$; bei Frauen beträgt die Differenz 50 \% zu 30 \%."

Zahlreiche weitere Indikatoren belegen, dass das Rauchen in unterschiedlichen Bevölkerungsschichten unterschiedlich stark ausgeprägt ist. So raucht laut Mikrozensus 2005 ein Anteil von 33 \% aller Personen mit einem monatlichen Einkommen von bis zu $1.300 €$, während dies lediglich $20 \%$ der Personen mit einem monatlichen Einkommen von über $4.500 €$ tun. Insofern wäre jegliche Erfolg versprechende Maßnahme zur Raucherentwöhnung auch ein Möglichkeit zur Minderung sozialer Ungleichheiten in Bezug auf Gesundheitschancen.
Auf diesen Aspekt der Gesundheitsversorgung bezieht sich das SGB V unter der Überschrift Prävention und Selbsthilfe in $§ 20$ (1) wie folgt: „[...] Leistungen zur Primärprävention sollen den allgemeinen Gesundheitszustand verbessern und insbesondere einen Beitrag zur Verminderung sozial bedingter Ungleichheit von Gesundheitschancen erbringen." Eine Einstufung von die Gesundheit gefährdenden Lebens- und Konsumgewohnheiten, die gerade in einkommensschwächeren im Vergleich zu anderen Schichten überproportional ausgeprägt sind, als „Lifestyle“, lässt die entsprechenden Bemühungen zumindest zum Teil ins Leere laufen. Auch wenn Primärprävention als solche sicherlich gegenüber anderen Ansätzen zu bevorzugen ist, kann ein Rückzug auf diese letztlich das Erreichen des Ziels, soziale Ungleichheiten zu mildern, gefährden. Vor dem Hintergrund des Ergebnisses von Lightwood/Glantz (1995), dass die Raucherentwöhnung bei Jugendlichen weniger kosteneffektiv sei als bei Erwachsenen, scheint auch eine Konzentration von Anstrengungen allein auf jugendliche Raucher auch aus ökonomischer Sicht fragwürdig.

Auch bestimmt der $§ 23$ (1) des SGB V, dass ein Anspruch auf Versorgung mit Arzneimitteln besteht, wenn diese notwendig sind, „eine Schwächung der Gesundheit, die in absehbarer Zeit voraussichtlich zu einer Krankheit führen würde, zu beseitigen" (Satz 1) bzw. „einer Gefährdung der gesundheitlichen Entwicklung eines Kindes entgegenzuwirken" (Satz 2). Die gesundheitlichen Folgen des Rauchens während der Schwangerschaft bzw. des Passivrauchens durch Kinder sind gut erforscht (Cook, 1999). Insofern werden auch die in $§ 23$ vorgegebenen Ziele in Bezug auf eine Vermeidung einer zukünftigen Krankheit bzw. einer Gefährdung der gesundheitlichen Entwicklung von Kindern durch die Ausschlussregelungen des $§ 34$ in Teilen konterkariert. Wenn im SGB V als wichtige Ziele der Gesundheitsversorgung apostrophierte Aspekte wie das verringern sozialer Ungleichheiten sowie der Schutz der Gesundheit von Kindern und Jugendlichen durch das Rauchen gefährdet sind, gleichzeitig aber effektive Maßnahmen der Raucherentwöhnung aus der Erstattung explizit ausgeschlossen werden, ergibt sich ein nicht aufzulösender Widerspruch.

Es bleibt offen, welche Gründe den Gesetzgeber zum Ausschluss bestimmter Präparate von der Erstattungsfähigkeit bewogen haben. Der Gesetzestext bleibt eine Begründung im Wesentlichen schuldig. Um für alle Beteiligten, insbesondere für die Patienten, eine Orientierung im Hinblick auf die Entwicklung im Gesundheitswesen zu ermöglichen, wäre eine transparente Darlegung der Kriterien, die hier eine Ausnahme begründen, wünschenswert. Eine Kasuistik von Ausnahmetatbeständen ist zu vermeiden. Das Abweichen von einer in der jüngeren Zeit forcierten Hinwendung zur (Kosten-) Nutzen-Logik des HTA durch eine Konstruktion von Ausnahmetatbeständen im Arzneimittelbereich und Verweis auf den Lifestyle-Charakter einiger Präparate ist schwer nachvollziehbar. 


\section{Fazit}

Die vorliegende Arbeit behandelte die Ausnahmeregelungen des §34 SGB V vor dem Hintergrund des zunehmenden Einsatzes der Methoden des HTA zur Ressourcenallokation im Gesundheitswesen. Ziel des Einsatzes von Kosten-Nutzwert-Analysen ist die Maximierung der Wohlfahrt. Die in $\$ 34$ als Lifestyle-Präparate eingestuften Wirkstoffe zur Raucherentwöhnung weisen Kosten-NutzwertRelationen auf, die nach allgemeinen Standards als vorteilhaft gelten. Die Begründung für den Ausschluss der genannten Wirkstoffe war in einem Gutachten für das BMG (IGES et al., 2006) als „äußerst problematisch“ charakterisiert worden. Die Frage, welche Überlegungen hinter dem Ausschluss dieser Präparate standen, bleibt offen.

Der Ausschluss der Wirkstoffe zur Raucherentwöhnung lässt befürchten, dass begrenzten Einsparungen heute erhebliche Mehrausgaben in Zukunft gegenüberstehen. Die Erstattung von Interventionen, die allein bei der vergleichsweise wenig effektiven Motivation von Rauchern ansetzen, stellt eine Fehllenkung von Mitteln dar. Sollte der Ausschluss automatisch auch alle neu verfügbar werdenden Wirkstoffe betreffen, sind negative Folgen für die Erforschung weiterer Wirkstoffe zu erwarten.

Gleichzeitig werden heute schon wichtige Ziele der Gesundheitspolitik wie das Verringern sozialer Ungleichheiten oder der Schutz der Gesundheit von Kindern und Jugendlichen gefährdet. Vor diesem Hintergrund wäre eine systematische Begründung entsprechender Ausnahmetatbestände dringend erforderlich.

\section{Literatur}

Arrow, K.J. / Cropper, M.L. / Eads, G.C. / Hahn, R.W. / Lave, L.B. / Noll, R. G. / Portney, P.R. / Russell, M. / Schmalensee, R. / Smith V.K. / Stavins, R.N. (1996): Is there a role for benefit-cost analysis in environment, health, and safety regulation? Science 272: 221-222.

Cahill, K.L. / Stead, F. / Lancaster, T. (2007): Nicotine receptor partial agonists for smoking cessation. Cochrane Database of Systematic Reviews 1

Cook, D.G. / Strachan, D.P. (1999): Summary of effects of parental smoking on the respiratory health of children and implications for research. Thorax 54:357-366.

Devlin, N. / Parkin, D. (2003): Does NICE have a cost effectiveness threshold and what other factors influence its decisions? A discrete choice analysis. City University London, School of Social Sciences, Discussion Paper Series No. 03/01.

Dolan, P. (2000): The measurement of health-related quality of life for use in resource allocation decisions in health care. In A. J. Culyer und J. P. Newhouse (Hrsg.) Handbook of Health Economics, Kap. 32, S. 17231760.

Fiore, M.C. / Bailey, W.C. / Cohen, S.J. et al. (2000): A clinical practice guideline for treating tobacco use and dependence - A US Public Health Service report. Journal of the American Medical Association 283 (24): 3244-3254.
Frijters, P. / Shields, M.A. / Wheatley Price S. / Williams, J. (2006) Quantifying the Cost of Passive Smoking on Child Health: Evidence from Children's Cotinine Samples. IZA Discussion Paper No. 2219.

IGES / Cassel, D. / Wille, E. / WidO (2006): Steuerung der Arzneitmittelausgaben und Stärkung des Forschungsstandortes für die pharmazeutische Industrie. Gutachten für das Bundesministerium für Gesundheit.

Kulp, W. / Greiner, W. (2006): Gesundheitsökonomie und HTA. Bundesgesundheitsblatt 39 (3): 257 - 263.

Lightwood, J.M. / Glantz S.A. (1997): Short-term Economic and Health Benefits of Smoking Cessation Myocardial Infarction and Stroke. Circulation 96:1089-1096.

Peto, R. / Lopez, A. / Boreham, D. J. / Thun, M. (2006): Moartality from smoking in developed countries 1950 - 2000. Mackay, J. / Eriksen, M. / Shafey, O. (Hrsg.) The Tobacco Atlas, American Cancer Society.

National Institute for Clinical Excellence (2002): Technology Appraisal No. 39 - Guidance on the use of nicotine replacement therapy (NRT) and bupropion for smoking cessation.

Ruff, L. K. / Volmer, T. / Nowak, D. / Meyer, A. (2000): The economic impact of smoking in Germany. European Respiratory Journal 16: 385 - 390.

Rasch, A / Greiner, W. (2006): Effekte einer Erstattung der Kosten für Raucherentwöhnungsmaßnahmen durch die Krankenversicherung. Prävention und Gesundheitsförderung 4: 269 - 277.

Sachverständigenrat für die Konzertierte Aktion im Gesundheitswesen (2003): Finanzierung, Nutzerorientierung und Qualität. Gutachten 2003 Band II: Qualität und Versorgungsstrukturen.

Song, F. / Raftery, J. / Aveyard, P. / Hyde, C. / Barton, P. / Woolacott, N. (2002): Cost-Effectiveness of Pharmacological Interventions for Smoking Cessation: A Literature Review and a Decision Analytic Analysis. Medical Decision Making.22(5): 26-37.

Twardella, T. / Brenner, H. (2007): Effects of practitioner education, practitioner payment and reimbursement of patient's drug costs on smoking cessation in primary care: a cluster randomised trial. Tobacco Control 16: 15-21

Weisbrod, B. A. (1991): The Health Care Quadrilemma: An Essay on Technological Change, Insurance, Quality of Care, and Cost Containment. Journal of Economic Literature 29 (2) 523 $-552$

Wegner, C. / Gutsch, A. / Hessel, F. / Wasem, J. (2004): Rauchenattributable Produktivitätsausfallkosten in Deutschland - eine partielle Krankheitskostenstudie unter Zugrundelegung der Humankapitalmethode. Gesundheitswesen 66: 423 - 432.

\section{Fußnoten}

1 So muss bspw. Unfruchtbarkeit, die mit einer in vitro Fertilisation behoben werden kann, nicht als Krankheitszustand eingestuft werden.

2 Bei IGES (2006) werden diese Kosten mit etwa 10 Mio. $€$ angegeben, realistischer dürfte ein Vielfaches davon sein. Allerdings erreichen die Kosten keine die Finanzierbarkeit des Systems tangierende Größenordnung.

3 Auch wäre die Gefahr von „Mitnahmeeffekten“ als Begründung für den Ausschluss der Erstattungsfähigkeit ethisch problematisch.

4 Pressemitteilung des BMG vom 13. Februar 2007. 